\title{
Intersensory facilitation of reaction time
}

LENORE MORRELL DIVISION OF NEUROLOGY, STANFORD UNIVERSITY SCHOOL OF MEDICINE

Human reaction times (RTs) to a photic stimulus were found to be shortened by an after-coming auditory stimulus. The effect was linearly related to interstimulus interval; the observed range was 20-120 ms. Faster RTs were found for shorter intervals.

Prolonged reaction times (RTs) to the second of two closely spaced stimuli in different sense modalities have been reported whether the $S$ was instructed to respond to both stimuli or only to the second (Davis, 1959). The shorter the interstimulus interval, the greater the observed delay. However, the generality of this observation may be limited to particular experimental conditions. It has also been reported that visual extraneous stimuli presented just prior to an auditory signal to respond may facilitate RT (John, 1964).

Helson (1964) briefly reported some findings for a bisensory task, wherein the $S$ was instructed to respond only to the first of two stimuli. RTs were found to be facilitated at interstimulus intervals less than 35 msec. and prolonged at intervals greater than 50 msec. when compared to RT in the reaction signal presented singly. Using a different method, Hershenson (1962) reported facilitation effects upon RT with paired visual and auditory stimuli at intervals up to 65 msec.

Electrophysiological methods have indicated that neural inhibition and facilitation may be demonstrated at various levels of the neuraxis in processing polysensory inputs. The present investigation was undertaken to assess the relationship between behavior in a cross-modal task, following the RT model used by Helson, and the averaged cortical potentials evoked by compound stimuli at various interstimulus intervals. Only the behavioral data will be reported here. Stimuli

The photic stimulus consisted of a 10-msec. flash delivered to both eyes from a pair of Sylvania glow modulator tubes (R1131c). These were mounted in a specially constructed frame with a viewing hood. The tubes were behind diffusing glasses which were 1 in. from the S's eyes. Intensity of light measured $50 \mathrm{ft}$. $-\mathrm{L}$ with a Spectra Brightness spot meter. Ss were instructed to keep their eyes closed throughout the experiment. (This was done in part to minimize possible eye-blink artifacts from the simultaneous EEG recordings.) With the eyes closed, the brightness of the light, after dark adaptation, was well above threshold (approximately $2 \mathrm{log}$ units). The auditory stimulus was a click of $70 \mathrm{~dB}$ delivered from a tweeter placed $4 \mathrm{ft}$. directly in front of the S's head. A warning tone of $130 \mathrm{cps}$ and $20 \mathrm{msec}$. duration occurred from 1-2 sec. before each trial.

\section{Procedure}

Ss were seated in a lounge chair in a dark, sounddeadened room. Each was instructed to respond as rapidly as possible only to the flash by depressing a small switch lightly taped to his right hand. Six Ss were used with replications on other occasions for two of these Ss. Each session consisted of 252 trials with 36 each of seven stimulus conditions. Six of these consisted of flash followed by click at the following intervals after flash onset: $20 \mathrm{msec}, 40$ msec., 80 msec., $100 \mathrm{msec} .$, and $120 \mathrm{msec}$. The other condition was flash presented alone. The seven conditions were presented in quasi-random order with the constraint that no pattern was repeated on the immediately following trial. Intertrial times ranged between 4-8 sec. Ss ranged in age from 20-38. All Ss were given a 5-min. warm-up before each session, which both familiarized them with the task and established dark adaptation. In each series, a 10-min. rest was given at the halfway point.

All experimental parameters and their sequences were controlled by a LINC computer. A 14-channel DAS-100 system served for recording of all data on FM tape, including the visual and auditory stimulus event markers and the motor response pulse. RTs were measured to $1 \mathrm{msec}$. accuracy by means of a time interval measuring program on the LINC.

\section{Results}

Figure 1 represents the relationship between RT to flash averaged over Ss as a function of the interstimulus interval separating the flash from the following click. The points plotted are the means of each S's median RT for each condition. (Approxi-

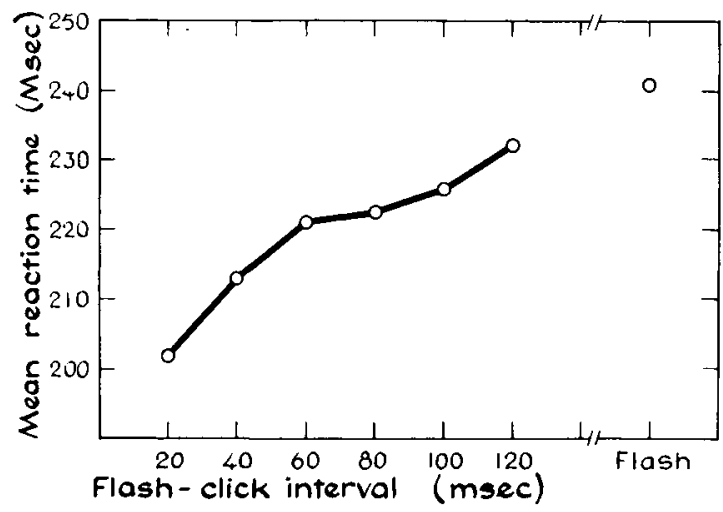

Fig. 1. Mean reaction time in relation to interstimulus interval; also, $\mathrm{RT}$ to flash alone $(\mathrm{N}=6)$. 
Table 1. Statisticall, wrumbant differences between RTs to the various milmulus configurations, testing all possible pairs. The T-method of multiple comparison was utilized (Scheffe', 1959). Single asterisk $\left(^{*}\right)$ indicates $5 \%$ significance level; double $\left(^{* *}\right)$, indicates $1 \%$ level.

\begin{tabular}{lcccccc} 
& $40 \mathrm{~ms}$ & $60 \mathrm{~ms}$ & $80 \mathrm{~ms}$ & $100 \mathrm{~ms}$ & $120 \mathrm{~ms}$ & $\begin{array}{c}\text { Flash } \\
\text { Alone }\end{array}$ \\
\hline $20 \mathrm{~ms}$ & - & $*$ & $*$ & $*$ & $* *$ & $* *$ \\
$40 \mathrm{~ms}$ & & - & - & - & $*$ & $*$ \\
$60 \mathrm{~ms}$ & & & - & - & - & $*$ \\
$80 \mathrm{~ms}$ & & & & - & - & $*$ \\
$100 \mathrm{~ms}$ & & & & - & - \\
$120 \mathrm{~ms}$ & & & & & - \\
\hline
\end{tabular}

mately 4 msec. ought to be added to each of the intervals to take into account the distance of the auditory source from S's ears.) Two-way analysis of variance showed that the treatment conditions were significant $(p=.005)$.

Table 1 shows the results of pair-wise comparisons (Scheffe, 1959) of RTs for the different stimulus conditions. Significant pair-wise differences were found between RT to flash alone on the one hand, and RT for stimuli with onset asynchronies ranging from 20 through $80 \mathrm{msec}$. Further, RT to flash-click at 20 msec. interstimulus interval was significantly shorter than that observed for all paired stimuli conditions of $60 \mathrm{msec}$. and longer. The only other significant pair-wise comparison was that between the $40-\mathrm{msec}$. pair and the 120-msec. pair.

Linear regression analysis of the grouped data was performed using only the paired stimuli data. RT to flash averaged over Ss is a linear function of the interstimulus interval between the flash and the following click. The slope of the function relating RT to interstimulus interval was $.27(p=.01)$. Subject by subject analysis indicates that individual slopes did not differ significantly from the group mean, although the intercept values did vary significantly among Ss.

All statistical analyses reported above were based only upon the first record obtained from a given S. These analyses were repeated so as to include the repeated run data (two additional runs for one $S$ and one additional run on another), and the results were unchanged.

\section{Discussion}

With the present stimuli, RT to a photic signal is facilitated by an auditory stimulus presented afterward.
The effect decrements with increasing temporal separation of the two stimuli. Pair-wise comparisons showed there are significant differences between RT to paired stimuli separated by as much as $80 \mathrm{msec}$. and RT to flash alone. These findings are thus at variance with those of Helson, who observed prolonged RTs at interstimulus intervals over $50 \mathrm{msec}$. when these were compared with single signal conditions. The many differences in both visual and auditory stimulus parameters, as well as experimental procedure, make analysis of the source of these differences difficult.

The present findings are similar to those reported by Reynolds (1966) in an unisensory task, which had the further feature that the Ss also responded to the second of the two visual stimuli. Reynolds also noted that the relationship between RT to the first of the two stimuli and interstimulus interval held under varying conditions of temporal and event uncertainty.

The observed linear relationship between RT and interstimulus interval and the obtained slope indicated that the Ss were not simply responding to the first signal perceived. Considering the differences in average latency of cortical arrival between visual and auditory stimuli, at $20 \mathrm{msec}$. asynchrony the click would arrive perhaps $20 \mathrm{msec}$. earlier than the flash, at $40 \mathrm{msec}$. the central arrival would be nearly simultaneous, and at all other intervals the flash would arrive first. If simple latency of arrival of "any signal" were operating, one would predict that only the 20 msec. pair would yield faster RTs when compared with all other conditions. Instead, the findings suggest that there is a facilitative process which shows gradual decrement with increasing temporal separation of the two stimuli.

\section{References}

Davis, $\mathbf{R}$. The role of "attention" in the psychological refractory period. Quart. J. exp. Psychol., 1959, 11, 211-220.

Helson, H. Current trends and issues in adaptation-level theory. Amer. Psychologist, 1964, 19, 26-38.

Hershenson, M. Reaction time as a measure of intersensory facilitation. J. exp. Psychol., 1962, 63, 289-293.

John. 1. D. The role of extraneous stimuli in responsiveness to signals: refractoriness or facilitation? Austral. J. Psychol., $1964,16,87-96$

Reynolds, D. Time and event uncertainty in unisensory reaction time. J. exp. Psychol., 1966, 71, 286-293.

Scheffe', H. The analysis of variance. New York: John Wiley \& Sons, 1959. P. 477. 